
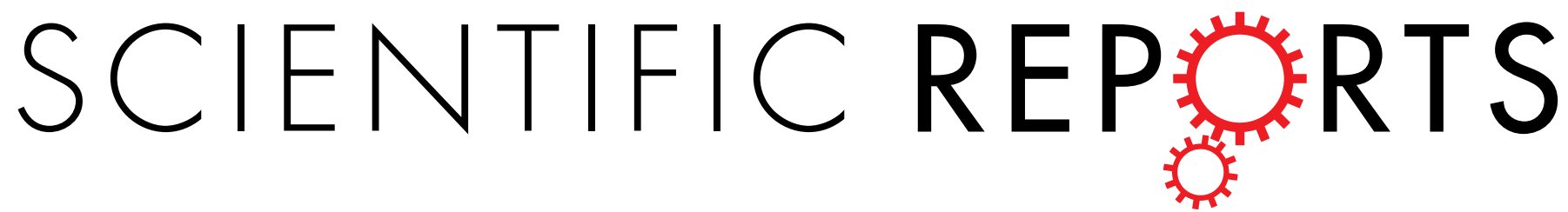

\title{
OPEN Association Between Helicobacter Pylori Infection and Long-term Outcome in Patients with Drug- \\ eluting Stent Implantation
}

Received: 31 October 2016

Accepted: 09 February 2017

Published: 13 July 2017

\author{
Rui Wang, Lei-lei Chen, De-zhao Wang \& Bu-xing Chen
}

To investigate the relationship between Helicobacter pylori $(\mathrm{Hp})$ infection and the long-term outcome in acute coronary syndrome (ACS) patients with drug-eluting stent (DES) implantation and so as to explore the significance of $\mathrm{Hp}$ eradication therapy in preventing major adverse cardiac events (MACE) and upper gastrointestinal bleeding (UGIB). 539 ACS patients with DES implantation from January 1, 2010 to December 31, 2012 were analyzed. All the patients were divided into two groups according to the result of ${ }^{13} \mathrm{C}$ urea breath test. 253 patients with $\mathrm{Hp}$ infection were put into group $\mathrm{A}\left(\mathrm{Hp}^{+}\right)$, and 286 cases without $\mathrm{Hp}$ infection were put into group $\mathrm{B}\left(\mathrm{Hp}^{-}\right)$. Demographic data was collected and all patients went through biochemical indicators and other routine blood examinations. We explored the correlations of Hp infection with MACE and UGIB after 3 to 5 years of follow-up using survival analysis. Survival analysis showed that Hp infection was a predictor of MACE and UGI. Sub-group analysis showed that patients with Hp eradication therapy had no relationship with MACE but had a lower rate of UGIB than those without Hp eradication therapy.

Helicobacter pylori ( $\mathrm{Hp})$ infection has been suspected as a pathogenic factor in many diseases. It is a frequent cause of dyspepsia, esophageal motility disorders ${ }^{1}$, gastro-esophageal reflux disease (GERD), peptic ulcer disease (PUD) and upper gastrointestinal bleeding (UGIB) ${ }^{2,3}$. As a precipitating factor of atherosclerotic plaque progression and instability, Hp infection may play a potential role in the pathogenesis of chest pain in patients with coronary artery disease $(\mathrm{CAD})^{4}$. Although there are still some doubts over the role of $\mathrm{Hp}$ infection in atherosclerosis, some epidemiological studies have shown that Hp seropositivity was significantly and positively associated with the occurrence of acute coronary syndrome (ACS) and atherosclerotic progression ${ }^{5}$. With the widespread use of drug-eluting stents (DES) in ACS patients, studies have shown that the incidence rate of UGIB was increasing year by year in the patients who receive dual anti-platelet treatment. The rates for patients taking and not taking dual antiplatelet drugs to develop UGIB are respectively $12.5 \%$ and $4.0 \%{ }^{6,7}$. Many ACS patients receiving dual anti-platelet therapy also suffer from $\mathrm{Hp}$ infection, which have greatly increased the incidence of UGIB. Major adverse cardiac events (MACE) is the primary end point, which includes all-cause mortality, myocardial infarction and target vessel revascularization. Previous studies have demonstrated that triple antiplatelet treatment after stent implantation was associated with feasible benefits on reducing the risk of MACE ${ }^{8}$

The Maastricht IV/Florence Consensus Report shows that those who taking dual anti-platelet drugs with $\mathrm{Hp}$ infection do not need the Hp eradication therapy if with no symptoms in digestive system ${ }^{9}$. However, it is still controversial over whether these asymptomatic patients with $\mathrm{Hp}$ infection should take eradication therapy or not, and this issue is not mentioned in the guideline. Radical treatment requires increased medication types and patient compliance. Whether Hp eradication therapy for patients with DES could reduce the UGIB and major adverse cardiac events (MACE) or not is still unclear.

The purpose of this study was to investigate the relationship between Hp infection and the long-term outcome of ACS patients who take dual anti-platelet therapy for one year after DES implantation, and the effects of $\mathrm{Hp}$ eradication therapy on the incidence of MACE and UGIB through a long-term follow-up. 


\section{Materials and Methods}

Subjects. Study design and setting. The following methods were carried out in accordance with the approved guidelines. All authors reviewed the results and approved the final version of the manuscript. This study was approved by the Ethics Committee of the Beijing Tiantan Hospital.

All experimental protocols were approved by Beijing Tiantan Hospital, and the written informed consent was obtaining from every patient. This study was a prospective, two-center trial. 572 ACS patients, who received DES implantation with dual antiplatelet therapy, hospitalized in Beijing Tiantan Hospital and Mentougou District Hospital from January 1, 2010 to December 31, 2012 were selected. Expect 33 patients lost to follow-up, a total of 539 patients with complete clinical data were collected in the study, and all the enrolled patients matched the inclusion and exclusion criteria. The disease history, physical examination, and laboratory results of patients were recorded. 539 subjects, with 184 males (34.1\%) and 355 females (65.9\%) were divided into two groups according to the result of ${ }^{13} \mathrm{C}$ urea breath test. 253 patients with $\mathrm{Hp}$ infection (positive of ${ }^{13} \mathrm{C}$ urea breath test) were set into group $\mathrm{A}\left(\mathrm{Hp}^{+}\right)$, and 286 cases without $\mathrm{Hp}$ infection were set into group $\mathrm{B}\left(\mathrm{Hp}^{-}\right)$. Group $\mathrm{A}\left(\mathrm{Hp}^{+}\right)$was further divided into two sub-groups (with/without Hp eradication therapy) using the digital random method.

Inclusion criteria. All the subjects met the following criteria: (1) with DES implantation following dual anti-platelet therapy; (2) having experienced ${ }^{13} \mathrm{C}$ urea breath test during hospitalization; and (3) having not received $\mathrm{Hp}$ eradication therapy before.

Exclusion criteria. Subjects who had suffered gastrointestinal bleeding, or with a history of gastrectomy, cardiac insufficiency, thyroid dysfunction, and any ongoing infections were eliminated. Patients who had used antibiotics, bismuth, proton pump inhibitor or sucralfate within one month were also excluded. Those who had gastrointestinal symptoms such as acid reflux, heartburn, nausea, vomiting, stomach ache and diarrhea, or had a confirmed peptic ulcer with Hp infection were also considered ineligible because they might have received gastroenterology treatment.

Tests and examinations. Routine examination. All patients underwent the following tests/examinations the next day after admission: blood routine examination, glycosylated hemoglobin, hs-CRP, HCY, blood coagulation, ${ }^{13} \mathrm{C}$ urea breath, and ultrasonic cardiogram (UCG). Subjects were asked to keep 8 hours of fasting for blood test.

${ }^{13} \mathrm{C}$ urea breath test. The ${ }^{13} \mathrm{C}$ urea breath test was conducted in the morning using the HCBT- 01 breath test automatic instrument (Shenzhen Zhonghe Haidewei Biological Technology Co. Ltd) and Hp infection was determined by ${ }^{13} \mathrm{C} /{ }^{12} \mathrm{C}$ isotope ratio $(\delta \%)$. Subjects were asked to achieve 2 hours of fasting, and specialized personnels were in strict accordance with the operating requirements. The positive value of Hp infection was defined as $\delta \geq 4 \pm 0.4 \%$.

Coronary angiography (CAG) and percutaneous coronary intervention ( $\mathrm{PCI})$. All patients underwent diagnostic CAG, which was performed based on the standard institution protocol, with 5 views of the left coronary, 2 views of the right coronary, and 2 orthogonal views of the target lesion. De-identified angiographic data sets were analyzed by a single interpreter. Minimal lumen diameter (MLD) was measured in the view with the greatest degree of stenosis. Proximal and distal reference diameters were measured and averaged to calculate the percentage of the diameter stenosis (\%DS).

All patients with acute ST-segment elevation myocardial infarction (STEMI) underwent therapeutic reperfusion using primary PCI. For all patients with non-ST-segment elevation myocardial infarction (NSTEMI) and unstable angina pectoris (UAP) the elective PCI was performed. Loading doses of asprin $(300 \mathrm{mg})$ and clopidogrel $(300 \mathrm{mg})$ were administered immediately after signing informed consent before taking PCI. Anticoagulation was achieved with unfractionated heparin $(100 \mathrm{IU} / \mathrm{kg})$, and an activated clotting time $\geq 250$ seconds was maintained. Aspiration thrombectomy, balloon angioplasty and stenting implantation were performed using standard techniques. Predilatation, direct stenting and post-stenting balloon inflation were performed at the operator's discretion. The procedure was considered successful if the residual stenosis was $<25 \%$ with a grade of 3 in Thrombolysis in Myocardial Infarction (TIMI) flow.

Eradication therapy. Eradication therapy was composed of rabeprazole ( $10 \mathrm{mg}$, twice daily), colloidal bismuth subcitrate ( $240 \mathrm{mg}$, twice daily), amoxicillin ( $1000 \mathrm{mg}$, twice daily), and clarithromycin ( $500 \mathrm{mg}$, twice daily) for ten days. If patients were allergic to penicillin, then amoxicillin was replaced with metronidazole ( $400 \mathrm{mg}$ twice daily). ${ }^{13} \mathrm{C}$ urea breath test should be performed at least for 4 weeks after the treatment ended, and patients should stop taking rabeprazole 2 weeks before the review for that false negative might be caused. If the initiative therapy failed, another regimen that was composed of rabeprazole (10 mg, twice daily), colloidal bismuth subcitrate ( $240 \mathrm{mg}$, twice daily), furazolidone ( $100 \mathrm{mg}$, twice daily) and tetracycline ( $500 \mathrm{mg}, 4$ times daily) for ten days would be selected as the rescue therapy.

Follow-up visits. All patients were followed up for 3 to 5 years at an outpatient clinic after hospital discharge. Follow-up visits with a cardiologist were scheduled 1 month after discharge and then every 6 month till the end of the study. During the follow-up, patients were monitored for MACE (including myocardial infarction, target vessel revascularization, and all-cause death) and UGIB, which were considered as end-points. Myocardial infarction (MI) was defined as chest pain with new ST-segment changes and elevation of cardiac markers which reflected myocardial necrosis to at least twice the upper limit of normal. PCI-related MI was not included as clinical events in this study. Target vessel revascularization (TVR) was defined as clinically driven percutaneous 
revascularization or bypass of the target lesion or any segment of the epicardial coronary artery that contained the target lesion. Otherwise, measurements were obtained at the end of the study. After completing data collection in all patients, we studied the association between the variables collected on admission and patient outcome after 3-5years.

Ethics. The study was approved by the Ethics Committee of Beijing Tiantan Hospital and Mentougou District Hospital. Informed consent was obtained for all the subjects and signed by themselves.

Statistical methods. Baseline characteristics of patients were compared using t-test for continuous variables and the chi-squared test for non-continuous variables. When continuous data was not normally distributed, groups were compared using nonparametric Wilcoxon rank sum test. The correlation between baseline characteristics and ACS was tested using a cox proportional hazards regression model. Clinically significant variables were adjusted and entered into a multivariate regression model using the method of backward stepwise: wald. $P<0.05$ was deemed statistically significant. Statistical analysis was performed using the SPSS software package (version 19.0).

\section{Results}

The baseline data of the two groups. There was no significant difference in the baseline data between group $\mathrm{A}\left(\mathrm{Hp}^{+}\right)$and $\mathrm{B}\left(\mathrm{Hp}^{-}\right)$(Table 1$)$. There were 126 patients receiving Hp eradication therapy. ${ }^{13} \mathrm{C}$ urea breath test was performed at least for 4 weeks after the treatment ended to estimate the therapeutic effects. The results of UBT test demonstrated that the initiative therapy was effective for the 126 patients, and none of them needed the rescue therapy.

Comparison of MACE and UGIB between the two groups. There were no significant differences in the prevalence of $\mathrm{MI}$ and revascularization between group $\mathrm{A}\left(\mathrm{Hp}^{+}\right)$and $\mathrm{B}\left(\mathrm{Hp}^{-}\right)$. But Group $\mathrm{A}\left(\mathrm{Hp}^{+}\right)$had more MACE and UGIB than group B $\left(\mathrm{Hp}^{-}\right)(17.4 \%$ vs. $9.1 \%, P=0.004 ; 13.4 \%$ vs. $5.2 \%, P=0.001)$ (Table 2 , Figs 1 and 2). There were no significant differences in the prevalence of death, MI and revascularization in sub-groups (Table 3 and Fig. 3). However, Hp + no therapy group had more UGIB than Hp + therapy group ( $18.1 \%$ vs. 8.7\%, $P=0.029$ ) (Table 3 and Fig. 4).

Muti-factorial analysis using the Cox proportional hazard model in patients with MACE and UGIB. In the multivariate Cox regression model, after adjusting for other important covariates, Hp infection remained an independent predictor for both MACE and UGIB (harzard ratio (HR) $=2.010,95 \%$ confidence interval $(95 \% \mathrm{CI})=1.109-3.645 ; \mathrm{HR}=2.609 ; 95 \% \mathrm{CI}=1.419-4.795$ (Table 4). In the sub-groups, Hp infection + no therapy was an independent predictor for MACE and UGIB (HR $=3.437,95 \% \mathrm{CI}=1.461-8.809$; $\mathrm{HR}=2.607,95 \%$ CI: 1.246-5.455) (Table 5).

\section{Discussion}

In this study, we first evaluated the effects of $\mathrm{Hp}$ infection and conventional cardiovascular disease risk factors on the incidence of future MACE among 539 patients presenting with ACS. The results reflected a strong association between Hp infection and the incidence of MACE in participants during the 3 to 5 years of follow-up after hospital discharge. However, we did not find any significant association between Hp infection and other studied risk factors. The statistically significant effect on the MACE in ACS patients was confirmed in survival analysis (Fig. 1) and multi-factorial analyses (Table 4). Sub-group analysis showed that patients with Hp eradication therapy had no relationship with MACE compared with those without Hp eradication therapy.

Our findings were consistent with majority of the previous studies, which also involved mixed subject groups, such as patients with normal coronary angiogram or significant narrowing of the coronary arteries. Significant correlation of Hp infection with ACS and cardiac syndrome X has ever been demonstrated in previous studies ${ }^{7-17}$, but contrary results have also been reported in other researches ${ }^{18-20}$. Hp is a pathogen that can cause persistent infection even be life-long, and antibodies could be persistently generated. Several pathological mechanisms have been postulated to explain the effect of $\mathrm{Hp}$ infection in atherosclerosis. It has been suggested that Hp initiates an acute-phase response and activates TNF- $\alpha$, IL- 6 and fibrinogen inflammatory cytokines, which could directly or indirectly propagate an inflammatory process in the arterial walls ${ }^{21,22}$. In addition, it can cause platelet aggregation, an important aspect of acute destabilization of atherosclerotic disease ${ }^{23}$. Hp may also lead to endothelial damage though aggravated autoimmune hormonal responses caused by antigenic mimicry ${ }^{24}$ as well as the immunological cross reactivity between bacterial and human heat-shock proteins ${ }^{25}$. This process may lead to coronary calcification and atherosclerosis ${ }^{26}$. In addition, researcher have found $\mathrm{Hp}$ in the arterial vascular wall ${ }^{19}$, and in the atherosclerotic plaque using polymerase chain reaction (PCR) technique ${ }^{27}$. Despite some conflicting data has been reported ${ }^{28}$, we considered that the discrepancy may be due to differences in the study populations and detection method ${ }^{29}$. In our study, we used ${ }^{13} \mathrm{C}$ urea breath test to detect $\mathrm{Hp}$ infection.

The study of Eskandarian et al. ${ }^{29}$ showed that Hp IgA seropositivity was significantly associated with fatal cardiovascular events, with a hazard ratio of 2.58 , which is similar to the findings in our study. As mentioned above, $\mathrm{Hp}$ has been shown to cause platelet aggregation ${ }^{23}$. During the acute phase of ACS and plaque disruption, this could lead to local inflammation and aggravated platelet aggregation, which is a crucial cause to acute myocardial ischemia. This process may explain why Hp infection was associated with long-term outcomes. Additionally, our results showed that no significant difference on MACE incidence was found between the Hp-positive patients with eradication therapy and $\mathrm{Hp}$-positive patients without eradication therapy $(14.3 \%$ vs. $20.5 \%, P=0.194$; Table 3 and Fig. 3). Therefore, it was concluded that Hp eradication therapy could not reduce the incidence of MACE. Moreover, this study also demonstrated a positive association between Hp infection and the incidence of UGIB in the participants during follow-up. It was statistically significant, as confirmed in the survival analysis 


\begin{tabular}{|c|c|c|c|}
\hline & Group A $\left(\mathrm{Hp}^{+}\right) \mathrm{n}=253$ & Group B $\left(\mathrm{Hp}^{-}\right) \mathrm{n}=286$ & $P$ value \\
\hline Females, n (\%) & $160(63.2)$ & $195(68.4)$ & 0.227 \\
\hline Smoke, n (\%) & $129(49.0)$ & $143(50.0)$ & 0.819 \\
\hline Hypertension, $\mathrm{n}(\%)$ & $135(53.4)$ & $147(51.4)$ & 0.649 \\
\hline Dyslipedemia, n (\%) & $104(41.1)$ & $103(36.0)$ & 0.225 \\
\hline Diabetes Melitus, n (\%) & $74(29.2)$ & $74(25.9)$ & 0.381 \\
\hline Cerebrovascular disease, $\mathrm{n}(\%)$ & $21(8.3)$ & $31(10.8)$ & 0.319 \\
\hline Family history of CAD, n (\%) & $16(6.3)$ & $13(4.5)$ & 0.361 \\
\hline Fundus abnormal, n (\%) & $225(88.9)$ & $249(87.1)$ & 0.799 \\
\hline Coronary artery lesions, $\mathrm{n}(\%)$ & & & 0.882 \\
\hline Single vessel lesion & $105(41.5)$ & $124(43.4)$ & \\
\hline Double or left main lesion & $71(28.1)$ & $80(28.0)$ & \\
\hline Triple vessel lesion & $77(30.4)$ & $82(28.7)$ & \\
\hline ACS, n (\%) & & & 0.425 \\
\hline UAP & $162(64.0)$ & $198(69.2)$ & \\
\hline AMI & $91(36.0)$ & $88(30.8)$ & \\
\hline Primary PCI, n (\%) & $87(34.4)$ & $94(32.9)$ & 0.709 \\
\hline Asprin, n (\%) & $249(98.4)$ & $283(99.0)$ & 0.870 \\
\hline Clopidogrel, n (\%) & $251(99.2)$ & $281(98.3)$ & 0.549 \\
\hline Mean age \pm SD (years) & $61.3 \pm 11.32$ & $60.6 \pm 10.80$ & 0.411 \\
\hline Mean stent $\pm \mathrm{SD}(\mathrm{mm})$ & $35.7 \pm 14.45$ & $37.9 \pm 15.10$ & 0.166 \\
\hline Mean SBP \pm SD $(\mathrm{mmHg})$ & $134.7 \pm 21.93$ & $137.8 \pm 23.85$ & 0.114 \\
\hline Mean $\mathrm{HR} \pm \mathrm{SD}$ (beats/min) & $74.2 \pm 11.72$ & $74.9 \pm 12.98$ & 0.458 \\
\hline Mean $\mathrm{WBC} \pm \mathrm{SD}\left(10^{9} / \mathrm{L}\right)$ & $6.9 \pm 2.71$ & $7.1 \pm 2.65$ & 0.537 \\
\hline Mean LVEF \pm SD (\%) & $60.9 \pm 7.50$ & $61.1 \pm 7.41$ & 0.874 \\
\hline Mean $\mathrm{BMI} \pm \mathrm{SD}\left(\mathrm{kg} / \mathrm{m}^{2}\right)$ & $24.7 \pm 3.52$ & $25.1 \pm 3.51$ & 0.094 \\
\hline $\begin{array}{l}\text { Median hs-CRP }(\mathrm{mg} / \mathrm{L}) \\
\text { (interquartile range) }\end{array}$ & $2.90(1.58-8.45)$ & $2.06(1.20-5.77)$ & 0.202 \\
\hline $\begin{array}{l}\text { Median HCY (umol/L) } \\
\text { (interquartile range) }\end{array}$ & $12.00(8.56-18.60)$ & $11.2(6.66-17.20)$ & 0.075 \\
\hline
\end{tabular}

Table 1. Baseline data of the two groups. Abbreviations: CAD, coronary artery disease; ACS, acute coronary syndrome; UAP, unstable angina pectoris; AMI, acute myocardial infarction; PCI, percutaneous coronary intervention; SD, standard deviation; SBP, systolic blood pressure; HR, heart rate; WBC, white blood-cell count; LVEF, left ventricular ejection fraction; BMI, body mass index; hs-CRP, high sensitive C-reactive protein; HCY, homocysteine.

\begin{tabular}{|c|c|c|c|}
\hline & $\begin{array}{c}\text { Group A }\left(\mathrm{Hp}^{+}\right) \\
\mathrm{n}=253\end{array}$ & $\begin{array}{c}\text { Group B }\left(\mathrm{Hp}^{-}\right) \\
\mathrm{n}=286\end{array}$ & $P$ value \\
\hline \multicolumn{4}{|l|}{ Follow-up (cumulated events), n (\%) } \\
\hline Death & $26(10.3)$ & $12(4.2)$ & 0.006 \\
\hline MI & $17(6.7)$ & $10(3.5)$ & 0.087 \\
\hline Revascularization & $12(4.7)$ & $6(2.1)$ & 0.088 \\
\hline MACE (death/MI/revascularization) & $44(17.4)$ & $26(9.1)$ & 0.004 \\
\hline UGIB, n (\%) & $34(13.4)$ & $15(5.2)$ & 0.001 \\
\hline
\end{tabular}

Table 2. Clinical events from PCI until end of follow up by two groups. Abbreviations: MI. myocardial infarction; MACE, major adverse cardiac events; UGIB, upper gastrointestinal bleeding.

(Fig. 2) and the multi-factorial analyses (Table 4). The Hp positive subjects without eradication therapy were at a 2.607-fold greater risk for UGIB than subjects with eradication therapy. Subjects who were Hp-positive with eradication therapy had significantly different outcome compared to the subjects who were Hp-positive without eradication therapy $(18.1 \%$ vs. $8.7 \%, P=0.029)$ (Table 3 and Fig. 4$)$.

In our study, no significant association was found between the conventional cardiac risk factors (hypertension, DM, hyperlipidemia, smoking, and positive family history) and the incidence of MACE. This was consistent with the results reported by Eskandarian et al., who did not demonstrate a prognostic role of these classic risk factors on MACE in patients with ACS either ${ }^{29}$. These results may be partly due to aggressive treatment of these risk factors in patients diagnosed with ACS in the study population. Longer follow-up duration and a larger number of sample populations may be needed in future to further evaluate the prognostic effects of these factors. 


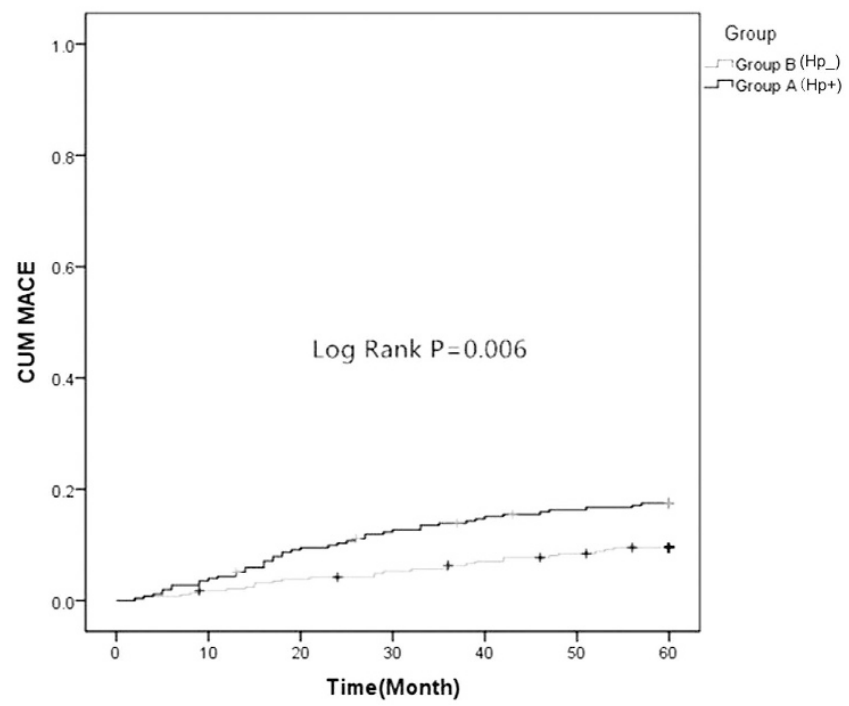

Figure 1. Comparison of two Kaplan-Meier curves as a function of time to the MACE between group A $\left(\mathrm{Hp}^{+}\right)$and group $\mathrm{B}\left(\mathrm{Hp}^{-}\right)(\log$ Rank test, $\mathrm{F}=7.699, P=0.006)$.

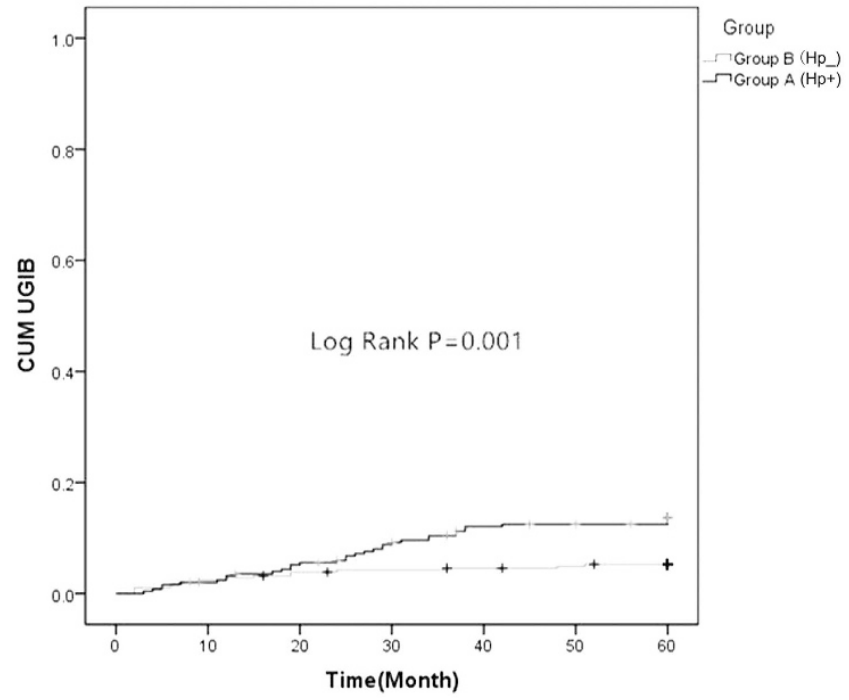

Figure 2. Comparison of two Kaplan-Meier curves as a function of time to the UGIB between group A $\left(\mathrm{Hp}^{+}\right)$and group $\mathrm{B}\left(\mathrm{Hp}^{-}\right)(\log$ Rank test, $\mathrm{F}=10.852, \mathrm{P}=0.001)$.

\begin{tabular}{|c|c|c|c|}
\hline & Hp + Therapy $n=126$ & Hp + No Therapy $n=127$ & $P$ value \\
\hline \multicolumn{4}{|l|}{ Follow-up (cumulated events), n (\%) } \\
\hline Death & $11(8.7)$ & $14(11.0)$ & 0.541 \\
\hline MI & $9(7.1)$ & $8(6.3)$ & 0.257 \\
\hline Revascularization & $4(3.2)$ & $9(7.1)$ & 0.159 \\
\hline MACE (death/MI/revascularization) & $18(14.3)$ & $26(20.5)$ & 0.194 \\
\hline UGIB, n (\%) & $11(8.7)$ & $23(18.1)$ & 0.029 \\
\hline
\end{tabular}

Table 3. Clinical events from PCI until end of follow up by sub-groups. Abbreviations: MI. myocardial infarction; MACE, major adverse cardiac events; UGIB, upper gastrointestinal bleeding; Hp+Therapy, Hp positive patients with $\mathrm{Hp}$ eradication therapy; $\mathrm{Hp}+$ No Therapy, $\mathrm{Hp}$ positive patients without Hp eradication therapy.

Earlier studies have shown that history of peptic ulcer, cardiogenic shock, cardiac arrest, inotropic agent requirement, and primary PCI are important risk factors for UGIB occurrence in patients with $\mathrm{CAD}^{4,30}$. However, the $\mathrm{Hp}$ infection was not shown to be an important risk factor for UGIB in patients with ACS in these studies ${ }^{4,31}$. 


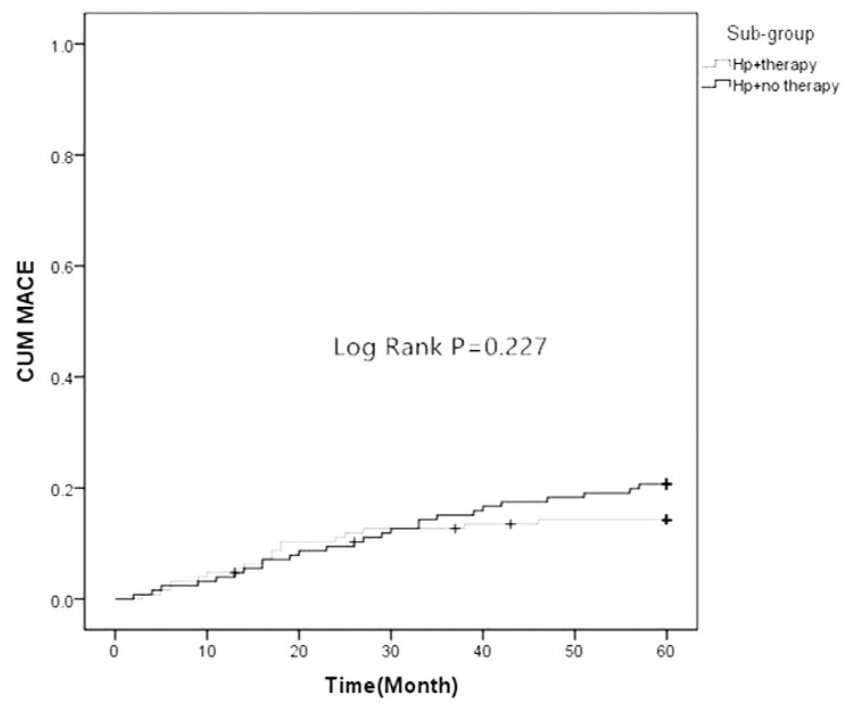

Figure 3. Comparison of two Kaplan-Meier curves as a function of time to the MACE between Hp-positive patients with eradication therapy $(\mathrm{Hp}+$ Therapy) and $\mathrm{Hp}$-positive patients without eradication therapy $(\mathrm{Hp}+$ No Therapy) $(\log$ Rank test, $\mathrm{F}=1.460, P=0.227)$.

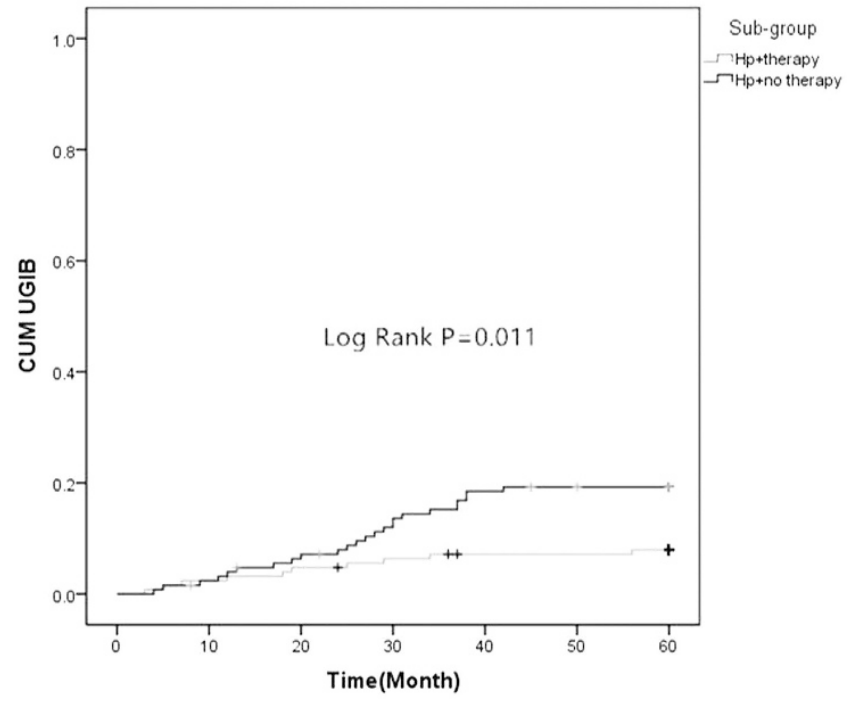

Figure 4. Comparison of two Kaplan-Meier curves as a function of time to the UGIB between Hp-positive patients with eradication therapy $(\mathrm{Hp}+$ Therapy) and $\mathrm{Hp}$-positive patients without eradication therapy $(\mathrm{Hp}+$ No Therapy) (Log Rank test, $\mathrm{F}=6.469, P=0.011)$.

\begin{tabular}{|c|c|c|c|c|c|}
\hline Category & B value & SE & Wald X ${ }^{2}$ & $P$ value & HR $(95 \% C I)$ \\
\hline \multicolumn{6}{|l|}{ MACE } \\
\hline Hp infection & 0.698 & 0.304 & 5.287 & 0.021 & $2.010(1.109-3.645)$ \\
\hline \multicolumn{6}{|l|}{ UGIB } \\
\hline HCY (umol/L) & 0.023 & 0.012 & 3.490 & 0.062 & $1.023(0.999-1.047)$ \\
\hline Hp infection & 0.959 & 0.311 & 9.534 & 0.002 & $2.609(1.419-4.795)$ \\
\hline
\end{tabular}

Table 4. Cox regression analysis in patients with MACE and UGIB (Method: Backwrd Stepwise-Wald). Abbreviations: MACE, major adverse cardiac events; UGIB, upper gastrointestinal bleeding; Hp, Helicobacter pylori; HCY, homocysteine; SE, standard error; HR, hazard ratio; CI, confidence interval. 


\begin{tabular}{|l|c|c|c|c|c|}
\hline Category & B value & SE & Wald X & P value & HR (95\%CI) \\
\hline \multicolumn{7}{|c|}{ MACE } \\
\hline Hp infection+no therapy & 1.235 & 0.437 & 7.994 & 0.005 & $3.437(1.461-8.809)$ \\
\hline LVEF & 0.079 & 0.028 & 7.719 & 0.005 & $1.082(1.023-1.143)$ \\
\hline \multicolumn{7}{|c|}{ UGIB } \\
\hline Hp infection+no therapy & 0.958 & 0.377 & 6.467 & 0.011 & $2.607(1.246-5.455)$ \\
\hline
\end{tabular}

Table 5. Cox regression analysis in the sub-groups patients with MACE and UGIB (Method: Backwrd Stepwise-Wald). Abbreviations: MACE, major adverse cardiac events; UGIB, upper gastrointestinal bleeding;LVEF, left ventricular ejection fraction; Hp, Helicobacter pylori; SE, standard error; HR, hazard ratio; $\mathrm{CI}$, confidence interval.

Hp infection is an important risk factor for peptic ulcer bleeding in those who receive aspirin therapy ${ }^{32}$. Our study has shown that Hp infection was an independent risk factor for UGIB in ACS patients after DES implantation with dual antiplatelet therapy, and Hp eradication therapy could reduce the incidence of UGIB. The divergence between the results of our study and previous studies may due to differences in the selected populations, study design, and variations in the follow-up. The molecular mechanism about the Hp eradication therapy reducing the incidence of UGIB in ACS patients with DES will be studied in the future.

\section{Conclusion}

The results obtained in this study have shown that the ACS patients with Hp infection had more coronary events and UGIB in the first 3 to 5 years after DES implantation. Hp eradication therapy could reduce the incidence of UGIB.

\section{Limitation}

The limitation of our study included relatively smaller sample size and less rigorous inclusion criteria. In addition, not all patients except those with UGIB required endoscopic follow-up.

\section{References}

1. Budzynski, J. et al. Autonomic nervous function in Helicobacter pylori-infected patients with atypical chest pain studied by analysis of heart rate variability. Eur J Gastroenterol Hepatol 16, 451-7 (2004).

2. Fashner, J. \& Gitu, A. C. Common Gastrointestinal Symptoms: dyspepsia and Helicobacter pylori. FP Essent 413, 24-8 (2013).

3. Roesler, B. M., Rabelo-Goncalves, E. M. \& Zeitune, J. M. Virulence Factors of Helicobacter pylori: A Review. Clin Med Insights Gastroenterol 7, 9-17 (2014).

4. Rogha, M. et al. Is helicobacter pylori infection a risk factor for coronary heart disease? ARYA Atheroscler 8, 5-8 (2012).

5. Vafaeimanesh, J. et al. Association of Helicobacter pylori infection with coronary artery disease: is Helicobacter pylori a risk factor? Scientific WorldJournal 2014, 516354 (2014).

6. Ng, F. H. et al. Gastrointestinal bleeding in patients receiving a combination of aspirin, clopidogrel, and enoxaparin in acute coronary syndrome. Am J Gastroenterol 103, 865-71 (2008).

7. Huang, K. W. et al. Risk factors for upper gastrointestinal bleeding in coronary artery disease patients receiving both aspirin and clopidogrel. J Chin Med Assoc 76, 9-14 (2013).

8. Fan, Z. G. et al. The clinical outcomes of triple antiplatelet therapy versus dual antiplatelet therapy for high-risk patients after coronary stent implantation: a meta-analysis of 11 clinical trials and 9,553 patients. Drug Des Devel Ther 10, 3435-3448 (2016).

9. Malfertheiner, P. et al. Management of Helicobacter pylori infection-the Maastricht IV/Florence Consensus Report. Gut 61, 646-64 (2012).

10. Hamm, C. W. et al. ESC Guidelines for the management of acute coronary syndromes in patients presenting without persistent STsegment elevation: The Task Force for the management of acute coronary syndromes (ACS) in patients presenting without persistent ST-segment elevation of the European Society of Cardiology (ESC). Eur Heart J 32, 2999-3054 (2011).

11. Wright, R. S. et al. 2011 ACCF/AHA focused update of the Guidelines for the Management of Patients with Unstable Angina/NonST-Elevation Myocardial Infarction (updating the 2007 guideline): a report of the American College of Cardiology Foundation/ American Heart Association Task Force on Practice Guidelines developed in collaboration with the American College of Emergency Physicians, Society for Cardiovascular Angiography and Interventions, and Society of Thoracic Surgeons. J Am Coll Cardiol 57, 1920-59 (2011).

12. Alpert, J. S., Thygesen, K., Antman, E. \& Bassand, J. P. Myocardial infarction redefined-a consensus document of The Joint European Society of Cardiology/American College of Cardiology Committee for the redefinition of myocardial infarction. J Am Coll Cardiol 36, 959-69 (2000).

13. Van de Werf, F. et al. Management of acute myocardial infarction in patients presenting with persistent ST-segment elevation: the Task Force on the Management of ST-Segment Elevation Acute Myocardial Infarction of the European Society of Cardiology. Eur Heart J 29, 2909-45 (2008).

14. Cutlip, D. E. et al. Clinical end points in coronary stent trials: a case for standardized definitions. Circulation 115, 2344-51 (2007).

15. Franceschi, F. et al. CagA antigen of Helicobacter pylori and coronary instability: insight from a clinico-pathological study and a meta-analysis of 4241 cases. Atherosclerosis 202, 535-42 (2009).

16. Celik, T., Iyisoy, A. \& Yuksel, U. C. Possible pathogenetic role of Helicobacter pylori infection in cardiac syndrome X. Int J Cardiol 142, 193-4 (2010).

17. Assadi, M. et al. The relation between Helicobacter pylori infection and cardiac syndrome X: a preliminary study. Int J Cardiol 134, e124-5 (2009).

18. Ozdogru, I. et al. The relationship between Helicobacter pylori IgG titre and coronary atherosclerosis. Acta Cardiol 62, 501-5 (2007).

19. Burke, C. A., Santisi, J., Church, J. \& Levinthal, G. The utility of capsule endoscopy small bowel surveillance in patients with polyposis. Am J Gastroenterol 100, 1498-502 (2005).

20. Delaney, B. C., Hobbs, F. D. \& Holder, R. Association of Helicobacter pylori infection with coronary heart disease. Eradication of the infection on grounds of cardiovascular risk is not supported by current evidence. BMJ 312, 251-2 (1996).

21. Sessa, R., Pietro, M. D., Filardo, S. \& Turriziani, O. Infectious burden and atherosclerosis: A clinical issue. World J Clin Cases 2, $240-9(2014)$. 
22. Tamer, G. S. et al. Helicobacter pylori seropositivity in patients with acute coronary syndromes. Dig Dis Sci 54, 1253-6 (2009).

23. Fagoonee, S. et al. Potential link between Helicobacter pylori and ischemic heart disease: does the bacterium elicit thrombosis? Minerva Med 101, 121-5 (2010).

24. Rechcinski, T., Kasprzak, J. D., Chmiela, M., Krzeminska-Pakula, M. \& Rudnicka, W. Patients with unstable angina pectoris present increased humoral response against Helicobacter pylori in comparison with patients with aggravated dyspepsia. Acta Microbiol Pol 51, 339-44 (2002).

25. Strachan, D. P. Non-gastrointestinal consequences of Helicobacter pylori infection. Br Med Bull 54, 87-93 (1998).

26. Zhu, J. et al. Association of serum antibodies to heat-shock protein 65 with coronary calcification levels: suggestion of pathogentriggered autoimmunity in early atherosclerosis. Circulation 109, 36-41 (2004).

27. Izadi, M. et al. Helicobacter species in the atherosclerotic plaques of patients with coronary artery disease. Cardiovasc Pathol 21, 307-11 (2012).

28. Smieja, M. et al. Multiple infections and subsequent cardiovascular events in the Heart Outcomes Prevention Evaluation (HOPE) Study. Circulation 107, 251-7 (2003).

29. Eskandarian, R. et al. Prognostic role of Helicobacter pylori infection in acute coronary syndrome: a prospective cohort study. Cardiovasc J Afr 23, 131-5 (2012).

30. Ng, F. H. et al. Upper gastrointestinal bleeding in patients with aspirin and clopidogrel co-therapy. Digestion 77, 173-7 (2008).

31. Shalev, A. et al. Incidence, predictors and outcome of upper gastrointestinal bleeding in patients with acute coronary syndromes. Int J Cardiol 157, 386-90 (2012).

32. Mousavi, M. et al. Relationship between continuous use of low-dose enteric-coated aspirin and gastrointestinal injuries in patients with gastrointestinal hemorrhage. Turk J Gastroenterol 24, 93-8 (2013).

\section{Author Contributions}

R.W. conceived and designed the experiments; L.C. conceived and performed the experiments; D.W. prepared figures. B.C. wrote the main manuscript text. All authors reviewed the manuscript.

\section{Additional Information}

Competing Interests: The authors declare no competing financial interests.

How to cite this article: Wang, R. et al. Association Between Helicobacter Pylori Infection and Long-term Outcome in Patients with Drug-eluting Stent Implantation. Sci. Rep. 7, 44954; doi: 10.1038/srep44954 (2017).

Publisher's note: Springer Nature remains neutral with regard to jurisdictional claims in published maps and institutional affiliations.

(c) (i) This work is licensed under a Creative Commons Attribution 4.0 International License. The images or other third party material in this article are included in the article's Creative Commons license, unless indicated otherwise in the credit line; if the material is not included under the Creative Commons license, users will need to obtain permission from the license holder to reproduce the material. To view a copy of this license, visit http://creativecommons.org/licenses/by/4.0/

(C) The Author(s) 2017 